\title{
On bornology of extended quasi-metric spaces
}

\author{
Olivier Olela Otafudu*1 (D), Wilson B. Toko ${ }^{1}$ (D), Danny Mukonda ${ }^{1,2}$ (D) \\ ${ }^{1}$ School of Mathematics, University of the Witwatersrand, Johannesburg 2050, South Africa \\ ${ }^{2}$ Department of Mathematics, Rusangu University, 660391, Monze, Zambia
}

\begin{abstract}
Beer studied the structure of sets equipped with the extended metrics with a focus on bornologies. In the paper [A. Piekosz and E. Wajch, Quazi-metrizability of bornological biuniverses inZF, J. Convex Anal. 2015], Piekosz and Wajch extended the well-known Hu's Theorem on boundedness in a topological space (see [S.-T. Hu, Boundedness in a topological space, J. Math. Pures Appl. 1949 ]) to the framework of quasi-metric spaces. In this note, we continue the work of Piekosz and Wajch. We show that many results on bornology of extended metric spaces due to Beer do not use the symmetry axiom of the extended metric, with appropriate modifications they still hold in the context of extended $T_{0}$-quasi-metric spaces.
\end{abstract}

Mathematics Subject Classification (2010). 54E35, 54E55, 54C35

Keywords. extended quasi-metric, metric bornology, universal space

\section{Introduction}

A bornology on a set $X$ is a collection $\mathscr{B}$ of subsets of $X$ which satisfies the following conditions:

(a) $\mathscr{B}$ forms a cover of $X$, i.e. $X=\bigcup \mathscr{B}$;

(b) for any $B \in \mathscr{B}$ and $A \subseteq B$, then $A \in \mathscr{B}$;

(c) $\mathscr{B}$ is stable under finite unions, i.e. if $X_{1}, X_{2}, \cdots, X_{n} \in \mathscr{B}$, then

$$
\bigcup_{i=1}^{n} X_{i} \in \mathscr{B} \text {. }
$$

If $\langle X, m\rangle$ is an extended metric space, then the bornology of $m$-bounded subsets of $X$ is denoted by $\mathscr{B}_{m}$ and it is called the metric bornology determined by the metric $m$.

In [1] Beer studied extended metric spaces and bornologies of these spaces. For instance, for a metrizable space $X$, he constructed an extended metric space $\left\langle W, \rho_{m}\right\rangle$ such that whenever $m$ is a compatible extended metric on $X$, one can find an isometry $\phi:\langle X, m\rangle \rightarrow$ $\left\langle W, \rho_{m}\right\rangle([1$, Theorem 3.2]).

Furthermore, he characterized the metric bornology $\mathscr{B}_{m}$ of an extended metric space $\langle X, m\rangle$ by using the well-know Hu's Theorem (see [3]) in the following ways:

\footnotetext{
*Corresponding Author.

Email addresses: olivier.olelaotafudu@wits.ac.za (O. Olela Otafudu),

Wilson.Toko@wits.ac.za (W.B. Toko), danny@aims.edu.gh (D. Mukondar)

Received: 29.01.2018; Accepted: 09.07.2018
} 
(1) Given a metrizable space $X$ and a bornology $\mathscr{B}$ on $X$, when does there exist an extended real-valued metric $m$ such that $\mathscr{B}=\mathscr{B}_{m}$ ?

(2) Given an extended metric $m$ on $X$, when does there exist a bona fide metric $m^{\prime}=\min \{1, m\}$ on $X$ such that $\mathscr{\mathscr { B }}_{m^{\prime}}=\mathscr{\mathscr { B }}_{m}$ ?

Piekosz and Wajch in [9] introduced the concepts of bornological biuniverse, properness and characteristic function in a bitopological space in the following way:

(a) A bornological biuniverse is an ordered pair $\left(\left(X, \tau_{1}, \tau_{2}\right), \mathscr{B}\right)$ where, $\left(X, \tau_{1}, \tau_{2}\right)$ is a bitopological space and $\mathscr{B}$ is a bornology on $X$.

(b) Let $\left(X, \tau_{1}, \tau_{2}\right)$ be a bitopological space. A boundedness $\mathscr{B}$ on $X$ is called $\left(\tau_{1}, \tau_{2}\right)$ proper if for each $A \in \mathscr{B}$, there exists $B \in \mathscr{B}$ such that $\operatorname{cl}_{\tau_{2}} A \subseteq \operatorname{int}_{\tau_{1}} B$.

(c) Let $\left(X, \tau_{1}, \tau_{2}\right)$ be a bitopological space. Then a $\left(\tau_{1}, \tau_{2}\right)$-characteristic function for a bornology $\mathscr{B}$ on $X$, is a bicontinuous function $f: X \rightarrow[0, \infty)$ such that $\mathscr{B}=\{A \subseteq X$ : $\sup \{f(x): x \in A\}<\infty\}$.

The following theorem is proved in [9].

Theorem 1.1. [9, Theorem 4.7] If $\left(X, \tau_{1}, \tau_{2}\right)$ is a quasi-metrizable bitopological space and $\mathscr{B}$ is a bornology on $X$, then the following conditions are equivalent:

(i) the bornological biuniverse $\left(\left(X, \tau_{1}, \tau_{2}\right), \mathscr{B}\right)$ is quasi-metrizable;

(ii) there exists a $\left(\tau_{1}, \tau_{2}\right)$-characteristic function for $\mathscr{B}$;

(ii) the bornology $\mathscr{B}$ is $\left(\tau_{1}, \tau_{2}\right)$-proper and it has a countable base.

The above theorem extended Hu's theorem from metric point of view to quasi-metrizable settings. Naturally this has led to the conjecture that Beer's results on bornology of extended metrics may also be investigated in the framework of extended quasi-metric spaces. The goal of this note is to give a careful study of the afore-mentioned conjecture.

\section{Preliminaries}

In this section we introduce the terminology and notation for quasi-metric spaces we will use in the sequel. Further details about theory of asymmetric topology can be found in [2] and [5].

Definition 2.1. Let $X$ be a set and let $q: X \times X \rightarrow[0, \infty)$ be a function mapping into the set $[0, \infty)$ of the nonnegative reals. Then $q$ is called a quasi-pseudometric on $X$ if

(a) $q(x, x)=0$ whenever $x \in X$,

(b) $q(x, z) \leq q(x, y)+q(y, z)$ whenever $x, y, z \in X$. Then pair $(X, q)$ is called quasipseudometric space.

We shall say that $q$ is a $T_{0}$-quasi-metric provided that $q$ also satisfies the following condition: For each $x, y \in X$,

$q(x, y)=0=q(y, x)$ implies that $x=y$. Then the pair $(X, q)$ is called $T_{0}$-quasi-metric space.

Remark 2.2. If we replace $[0, \infty)$ by $[0, \infty]$ (where for a $q$ attaining the value $\infty$ the triangle inequality is interpreted in the obvious way), then in such a case we shall speak of an $e x-$ tended quasi-pseudometric. If $X$ is a nonempty set and $q$ is an extended quasi-pseudometric on $X$, then the pair $\langle X, q\rangle$ is called extended quasi-pseudometric space. Moreover, if $q$ is an extended $T_{0}$-quasi-pseudometric on $X$, the pair $\langle X, q\rangle$ is called extended $T_{0}$-quasi-metric space. Note that if $q$ is an extended quasi-pseudometric on $X$ then $q^{-1}: X \times X \rightarrow[0, \infty]$ defined by $q^{-1}(x, y)=q(y, x)$ whenever $x, y \in X$ is also an extended quasi-pseudometric and $q^{s}=q \vee q^{-1}$ is an extended pseudometric on $X$. 
Let $\langle X, q\rangle$ be an extended quasi-pseudometric space. For each $x \in X$ and $\epsilon>0$, the set $B_{q}(x, \epsilon)=\{y \in X: q(x, y)<\epsilon\}$ denotes the open $\epsilon$-ball at $x$. The collection of all "open" balls yields a base for a topology $\tau(q)$. It is called the topology induced by $q$ on $X$. Similarly for each $x \in X$ and $\epsilon \geq 0$, we define the ball $C_{q}(x, \epsilon)=\{y \in X: q(x, y) \leq \epsilon\}$. Note that this latter set is $\tau\left(q^{-1}\right)$-closed, but not $\tau(q)$-closed in general.

A pair $\left(B_{q}(x, r) ; B_{q^{-1}}(x, s)\right)$ with $x \in X$ and nonnegative reals $r, s$ will be called a double ball at $x$.

Let us recall that a quasi-uniformity on a set $X$ is a filter $\mathcal{U}$ on $X \times X$ such that:

(i) for each $U \in \mathcal{U},\{(x, x): x \in X\} \subseteq U$;

(ii) for each $U \in \mathcal{U}$, there is $V \in \mathcal{U}$ such that $V^{2} \subseteq U$. Here $V^{2}=V \circ V=\{(x, z) \in$ $X \times X$ : there is $y \in X$ such that $(x, y) \in V$ and $(y, z) \in V\}$. A quasi-uniform space is a pair $(X, \mathcal{U})$ such that $X$ is a (nonempty) set and $\mathcal{U}$ is a quasi-uniformity on $X$.

Definition 2.3. [8, Definition 2.1] Let $(X, \mathcal{U})$ be a quasi-uniform space and $A \subseteq X$. The set $A$ is said to be bounded if for any $U \in \mathcal{U}$, there exists $n \in \mathbb{N}$ and a finite subset $F$ of $X$ such that $A \subseteq U^{n}[F]$, where

$$
U[F]=\bigcup_{f \in F} U(f)=\{y \in X: \text { there exists } f \in F \text { such that }(f, y) \in U\} .
$$

Remark 2.4. It has been observed in [8] that if a set is bounded in the sense of the above definition, then it is also bounded in the metric sense.

Let $\langle X, q\rangle$ be an extended quasi-metric space. Then for any $x, y \in X$, we define a relation $\mathcal{R}_{q}$ on $X$ by

$$
x \mathcal{R}_{q} y \text { provided } q(x, y)<\infty \text { and } q(y, x)<\infty .
$$

Remark 2.5. If $\langle X, q\rangle$ is an extended quasi-metric space, then the relation $\mathcal{R}_{q}$ is an equivalence relation on $X$.

Note that if $q=q^{-1}$ then the relation $\mathcal{R}_{q}$ is exactly the equivalence relation in sense of Beer (see [1]).

Let $x \in X$. Then the equivalence class of $x$ denoted by $\mathrm{qmc}_{q}(x)$ will be called quasimetric component of $x$.

Remark 2.6. For any extended $T_{0}$-quasi-metric space $\langle X, q\rangle$, it is easy to see that $\mathrm{qmc}_{q^{s}}(x) \subseteq \mathrm{qmc}_{q}(x)$ whenever $x \in X$. Here $\mathrm{qmc}_{q^{s}}(x)$ is a metric component in the sense of $[1]$.

Let $\langle X, q\rangle$ be an extended $T_{0}$-quasi-pseudometric space and let $x \in X$. Obviously $\mathrm{qmc}_{q}(x) \neq \emptyset$, since $x \in \mathrm{qmc}_{q}(x)$. We equip $\mathrm{qmc}_{q}(x)$ with the quasi-metric $q_{x}$ defined by $q_{x}:=\left.q\right|_{\mathrm{qmc}_{q}(x)}$. It follows that

$$
\tau(q)=\bigcup_{x \in X} \tau\left(q_{x}\right) \text { and } X=\bigoplus_{x \in X} \mathrm{qmc}_{q}(x) .
$$

Moreover, if $X$ can be partitioned into nonempty $\tau(q)$-clopen sets $\left\{\mathrm{qmc}_{q}(x): x \in X\right\}$ which are quasi-metrizable, then choosing a compatible quasi-metric $q_{x}$ for $\mathrm{qmc}_{q}(x)$ whenever $x \in X$, the extended $T_{0}$-quasi-metric $q$ can be defined by

$$
q(y, z)=\left\{\begin{array}{cc}
q_{x}(y, z) & \text { if }\{y, z\} \in \mathrm{qmc}_{q}(x) \text { for some } x \in X \\
\infty & \text { otherwise. }
\end{array}\right.
$$

The proof of the next lemma follows from the proof of [1, Proposition 2.3] since the symmetric axiom of a metric is not used.

Lemma 2.7. A quasi-metrizable space $X$ is connected if and only if each extended $T_{0^{-}}$ quasi-metric on $X$ is a $T_{0}$-quasi-metric. 


\section{Construction of the universal space}

Let $(X, q)$ be a quasi-pseudometric space. In the sequel, we set $\mathcal{C}(X, q)=\left\{\left(f_{1}, f_{2}\right): f_{1}\right.$ and $f_{2}$ are continuous real-valued functions on $\left.X\right\}$ and

$$
\mathcal{C}^{b}(X, q)=\left\{\left(f_{1}, f_{2}\right) \in \mathcal{C}(X, q): f_{1} \text { and } f_{2} \text { are bounded on }\left(X, q^{s}\right)\right\} .
$$

If $a, b \in \mathbb{R}$ we shall put $a \dot{-} b=\max \{a-b, 0\}$. We equip $\mathcal{C}(X, q)$ with the extended $T_{0}$-quasi-metric $Q_{q}$ defined by

$$
Q_{q}(f, g)=\sup _{x \in X}\left(f_{1}(x) \dot{-} g_{1}(x)\right) \vee \sup _{x \in X}\left(g_{2}(x) \dot{-} f_{2}(x)\right)
$$

whenever $f=\left(f_{1}, f_{2}\right), g=\left(g_{1}, g_{2}\right) \in \mathcal{C}(X, q)$ (see [4]).

Obviously, $\left(\mathrm{e}^{b}(X, q), Q\right)$ is a $T_{0^{-}}$quasi-metric space, when $Q=\left.Q_{q}\right|_{\mathfrak{e}^{b}(X, q)}$. Note that for a fixed $a \in X$ and for any $b \in X$, define the function pair $e_{X}(b)=\left(\left(e_{X}\right)_{1}(b),\left(e_{X}\right)_{2}(b)\right)$, where

$$
\left(e_{X}\right)_{1}(b)=q(b, x)-q(a, x)
$$

and

$$
\left(e_{X}\right)_{2}(b)=q(x, b)-q(x, a),
$$

whenever $x \in X$. It is clear that $\left(e_{X}\right)_{1}$ and $\left(e_{X}\right)_{2}$ are bounded by $q^{s}(a, b)$.

Then the map $e_{X}:\langle X, q\rangle \longrightarrow\left(\mathcal{C}^{b}(X, q), Q\right)$ defined by $b \mapsto e_{X}(b)$ yields an isometric embedding. Indeed, for any $b, b^{\prime} \in X$, it follows that

$$
\sup _{x \in X}\left[(q(b, x)-q(a, x)) \dot{-}\left(q\left(b^{\prime}, x\right)-d(a, x)\right)\right]=q\left(b, b^{\prime}\right)
$$

and

$$
\sup _{x \in X}\left[\left(q\left(x, b^{\prime}\right)-q(x, a)\right) \dot{-}(q(x, b)-d(x . a))\right]=q\left(b, b^{\prime}\right) .
$$

Therefore $Q\left(e_{X}(b), e_{X}\left(b^{\prime}\right)\right)=q\left(b, b^{\prime}\right)$.

We observe that

$$
Q^{s}(f, g)=\sup _{x \in X}\left|f_{1}(x)-g_{1}(x)\right| \vee \sup _{x \in X}\left|g_{2}(x)-f_{2}(x)\right|
$$

whenever $f=\left(f_{1}, f_{2}\right), g=\left(g_{1}, g_{2}\right) \in \mathrm{e}^{b}(X, q)$.

Let $\triangle_{q}(X, q)=\left\{(f ; A): f=\left(f_{1}, f_{2}\right) \in \mathcal{C}(A, q)\right.$ and $\left.A \in \mathscr{C}_{0}\left(X, q^{s}\right)\right\}$. Here, $\mathscr{C}_{0}\left(X, q^{s}\right)$ is the set of nonempty subsets of $X$ which are closed with respect to $\tau\left(q^{S}\right)$.

We define an extended $T_{0}$-quasi-metric $E_{X}$ on $\triangle_{q}(X, q)$ by

$$
E_{X}((f ; A),(g ; B))=\left\{\begin{array}{cl}
\infty & \text { if } A \neq B \\
\sup _{x \in A}\left(f_{1}(x) \dot{-} g_{1}(x)\right) \vee \sup _{x \in A}\left(g_{2}(x) \dot{-} f_{2}(x)\right) & \text { if } A=B .
\end{array}\right.
$$

Remark 3.1. We observe that

$$
\left(E_{X}^{s}\right)((f ; A),(g ; B))=\left\{\begin{array}{cc}
\infty & \text { if } A \neq B \\
\sup _{x \in A}\left|f_{1}(x)-g_{1}(x)\right| \vee \sup _{x \in A}\left|g_{2}(x)-f_{2}(x)\right| & \text { if } A=B .
\end{array}\right.
$$

Furthermore, $\left\langle\triangle_{q}(X, q), E_{X}^{s}\right\rangle$ is a complete extended metric space by [1, Proposition 3.1]. Therefore, the extended $T_{0}$-quasi-metric space $\left\langle\triangle_{q}(X, q), E_{X}\right\rangle$ is bicomplete by [4, Corollary 3].

Proposition 3.2. Let $(X, q)$ be a $T_{0}$-quasi-metric space. Then the map $\theta:\langle X, q\rangle \longrightarrow$ $\left\langle\triangle_{q}(X, q), E_{X}\right\rangle$ defined by $\theta(x)=\left(\left(\theta_{1}(x), \theta_{2}(x)\right) ; q m c_{q}(x)\right)$, where $\theta_{1}(x)=\left.q(x, \cdot)\right|_{q m c_{q}(x)}$ and $\theta_{2}(x)=\left.q(\cdot, x)\right|_{q m c_{q}(x)}$ whenever $x \in X$ is an isometry. Moreover, $\theta$ is injective. 
Proof. Let $x, y \in X$ such that $x \neq y$.

Case 1. If $q(x, y)=\infty$ and $q(y, x)=\infty$, then $\mathrm{qmc}_{q}(x) \neq \mathrm{qmc}_{q}(y)$. We have $E_{X}(\theta(x), \theta(y))=$ $\infty=q(x, y)$.

Case 2. If $q(x, y)=\infty$ and $q(y, x)<\infty$, then $\mathrm{qmc}_{q}(x) \neq \mathrm{qmc}_{q}(y)$. So $E_{X}(\theta(x), \theta(y))=$ $\infty=q(x, y)$.

Case 3. If $q(x, y)<\infty$ and $q(y, x)=\infty$, then case is similar to Case 2 .

Case 4. If $q(x, y)<\infty$ and $q(y, x)<\infty$, then

$$
\mathrm{qmc}_{q}(x)=\mathrm{qmc}_{q}(y) \text {. }
$$

One sees that

$$
\sup _{a \in \operatorname{qmc}_{q}(x)}(q(x, a)-q(y, a))=q(x, y)
$$

by letting $y=a$ and by the triangle inequality. Similarly,

$$
\sup _{a \in \mathrm{qmc}_{q}(x)}(q(a, y)-q(a, x))=q(x, y) .
$$

Thus, $E_{X}(\theta(x), \theta(y))=q(x, y)$. Hence $\theta$ is an isometry map. Since $\langle X, q\rangle$ is $T_{0}$, it follows that map $\theta$ is injective by [6, Lemma 4$]$.

Example 3.3. (compare [4, Remark 4]) Let $(X, q)$ be a $T_{0}$-quasi-metric space and let $\mathcal{P}_{0}(X)$ be the set of nonempty subsets of $X$. Then for any $A \in \mathcal{P}_{0}(X)$, set $\left(f_{A}\right)_{1}(x):=$ $\operatorname{dist}(A, x)$ and $\left(f_{A}\right)_{2}(x):=\operatorname{dist}(x, A)$ whenever $x \in X$. Then for the function pair $f_{A}=$ $\left(\left(f_{A}\right)_{1},\left(f_{A}\right)_{2}\right)$, we have $\left(f_{A}\right)_{1}:\left(X, q^{-1}\right) \longrightarrow(\mathbb{R}, u)$ is a nonexpansive map and $\left(f_{A}\right)_{2}$ : $(X, q) \longrightarrow(\mathbb{R}, u)$ is a nonexpansive map, where $u(x, y)=\max \{x-y, 0\}$ whenever $x, y \in \mathbb{R}$. Furthermore, $q_{H}(A, B)=Q_{q}\left(f_{A}, f_{B}\right)$ whenever $A, B \in \mathcal{P}_{0}(X)$, where $q_{H}(A, B)$ is the extended Hausdorff quasi-pseudometric on $\mathcal{P}_{0}(X)$.

Proposition 3.4. If $(X, m)$ is a metric space, then the map $\rho(f)=(f, f)$ defines an isometric embedding of $\left\langle\triangle_{m}(X, m), \rho_{X}\right\rangle$ into $\left\langle\triangle_{q}(X, m), E_{X}\right\rangle$.

Proof. Suppose that $(f ; A) \in \triangle_{m}(X, m)$. Then $((f, f) ; A) \in \triangle_{q}(X, m)$ as $(f, f)$ is a pair of continuous functions and $A$ is a nonempty $\tau(d)$-closed subset of $X$.

Let $((f, f) ; A),((g, g) ; B) \in \triangle_{q}(X, d)$. If $A=B$, then

$$
\begin{aligned}
\rho_{X}((f ; A),(g ; B)) & =\sup _{a \in A}|f(a)-g(a)| \\
& =\sup _{a \in A}(f(a) \dot{-} g(a)) \vee \sup _{a \in A}(g(a) \dot{-} f(a)) \\
& =E_{X}[((f, f) ; A),((g, g) ; B)] .
\end{aligned}
$$

If $A \neq B$, then obviously we have

$$
\rho_{X}((f ; A),(g ; B))=\infty=E_{X}[((f, f) ; A),((g, g) ; B)] .
$$

The following result extends Beer's result about the existence of a contravariant functor from the category of Hausdorff spaces and continuous surjective maps as morphisms and the category of spaces of partial maps (that is, continuous real valued functions restricted to the nonempty closed subsets) and isometric embedding maps as morphisms.

Theorem 3.5. Let $\left(X, q_{X}\right)$ and $\left(Y, q_{Y}\right)$ be two $T_{0}$-quasi-metric spaces.

If $\rho: X \rightarrow Y$ is a continuous surjective map, then there exists an isometry $\psi$ : $\left\langle\triangle_{q}\left(Y, q_{Y}\right), E_{Y}\right\rangle \rightarrow\left\langle\triangle_{q}\left(X, q_{X}\right), E_{X}\right\rangle$. 
Proof. Suppose that $A \subseteq Y$ and $f=\left(f_{1}, f_{2}\right) \in \mathcal{C}\left(A, q_{Y}\right)$. Then we define $\psi$ by

$$
\psi((f ; A))=\left[\left(\left.\left(f_{1} \circ \rho\right)\right|_{\rho^{-1}(A)},\left.\left(f_{2} \circ \rho\right)\right|_{\rho^{-1}(A)}\right) ; \rho^{-1}(A)\right] .
$$

We prove that $\psi$ is an isometry.

Case 1. If $A$ is $\tau\left(q_{Y}^{s}\right)$-closed subset of $Y$ and $f=\left(f_{1}, f_{2}\right), g=\left(g_{1}, g_{2}\right) \in \mathcal{C}\left(A, q_{Y}\right)$, then $\rho^{-1}(A)$ is $\tau\left(q_{X}^{s}\right)$-closed subset of $X$.

Since $\rho$ is surjective, we have

$$
\begin{aligned}
E_{X}(\psi((f ; A)), \psi((g ; A))) & =\sup _{x \in \rho^{-1}(A)}\left[\left(f_{1} \circ h\right)(x) \dot{-}\left(g_{1} \circ h\right)(x)\right] \vee \sup _{x \in \rho^{-1}(A)}\left[\left(g_{2} \circ h\right)(x) \dot{-}\left(f_{2} \circ h\right)(x)\right] \\
& =\sup _{y \in A}\left(f_{1}(y) \dot{-} g_{1}(y)\right) \vee \sup _{y \in A}\left(g_{2}(y) \dot{-} f_{2}(y)\right) \\
& =E_{Y}((f ; A),(g ; A)) .
\end{aligned}
$$

Case 2. Let $A_{1}$ and $A_{2}$ are two different $\tau\left(q_{Y}^{s}\right)$-closed subsets of $Y$. Then by continuity and surjectiveness of $\rho$ we have the sets $\rho^{-1}\left(A_{1}\right)$ and $\rho^{-1}\left(A_{2}\right)$ as the two different $\tau\left(q_{X}^{s}\right)$ closed subsets of $X$. Therefore,

$$
E_{X}\left(\psi\left(\left(f ; A_{1}\right)\right), \psi\left(\left(g ; A_{2}\right)\right)\right)=\infty=E_{Y}\left(\left(f ; A_{1}\right),\left(g ; A_{2}\right)\right)
$$

whenever $f=\left(f_{1}, f_{2}\right) \in \mathcal{C}\left(A_{1}, q_{Y}\right)$ and $g=\left(g_{1}, g_{2}\right) \in \mathcal{C}\left(A_{2}, q_{Y}\right)$.

\section{Bornology of quasi-metrically bounded subsets}

In what follows, we use the terminology of [9].

Definition 4.1. $[7$, p.4] Let $(X, q)$ be a quasi-pseudometric space. An arbitrary subset $A$ of $X$ is called q-bounded if and only if there exists $x \in X, r>0$ and $s>0$ such that $A \subseteq$ $B_{q}(x, r) \cap B_{q^{-1}}(x, s)$. Note that one can replace $B_{q}(x, r) \cap B_{q^{-1}}(x, s)$ by $C_{q}(x, r) \cap C_{q^{-1}}(x, s)$.

We recall that any the set of the form $B_{q}(x, r) \cap B_{q^{-1}}(x, s)$ is called double open ball.

We point out that Definition 4.1 is slightly different from [9, Definition 1.5]. In the sense of [9] a subset $A$ of $X$ can be $q$-bounded and not necessary $q^{-1}$-bounded. Obviously in our context a subset $A$ is $q$-bounded if and only if it is $q^{-1}$-bounded. Moreover, if $A$ is $q$-bounded, then $A$ is $q^{s}$-bounded too, but the converse is not true in general (see Remark 4.2 below).

Remark 4.2. [8, p.370] It is easy to see that if a set is $q^{s}$-bounded, then it is $q$-bounded but the converse is not true. Indeed let's equip $[0,1]$ with the $T_{0}$-quasi-metric

$$
q(x, y)=\left\{\begin{array}{cc}
y-x & \text { if } x \leq y \\
1 & x>y
\end{array}\right.
$$

and $\mathcal{U}$ is the quasi-uniformity generated by $q$ on $[0,1]$ and $\mathcal{U}^{-1}$ the conjugate quasiuniformity of $\mathcal{U}$ on $[0,1]$, then $([0,1], \mathcal{U})$ and $\left([0,1], \mathcal{U}^{-1}\right)$ are bounded quasi-uniform spaces but $\left([0,1], \mathcal{U}^{s}\right)$ is the discrete uniform space and it is not bounded.

One can easily show that Definition 4.1 is equivalent to Definition 4.3 below.

Definition 4.3. One says that a subset $A$ of a quasi-pseudometric space $(X, q)$ is $q$ bounded if there exist $\epsilon>0$ such that $q(x, y)<\epsilon$, whenever $x, y \in A$.

Definition 4.4. Let $(X, q)$ be a quasi-pseudometric space. Then $\mathscr{\mathscr { B }}_{q}(X)$ is the collection of all $q$-bounded subsets of $X$ in the sense of Definition 4.1.

As in metric settings, the collection $\mathscr{\mathscr { B }}_{q}(X)$ of $q$-bounded subsets of a quasi-pseudometric space $(X, q)$ forms a bornology on $X$. Then we call $\mathscr{\mathscr { B }}_{q}(X)$ the quasi-metric bornology determined by $q$. Moreover, we observe that $\mathscr{\mathscr { B }}_{q^{s}}(X)$ is a metric bornology determined by the metric $q^{s}$ in the sense of [1].

From Remark 4.2, we have the following observation. 
Lemma 4.5. Let $(X, q)$ be a quasi-metric space. Then the following statements are true:

(a) $\mathscr{\mathscr { B }}_{q^{s}}(X) \subseteq \mathscr{\mathscr { B }}_{q}(X)$,

(b) $\mathscr{\mathscr { O }}_{q^{s}}(X) \subseteq \mathscr{\mathscr { B }}_{q^{-1}}(X)$.

We recall that two bornologies on set are equivalent if they determine the same collection of bounded sets (see [1, p.3]).

Lemma 4.6. Let $(X, q)$ be a quasi-metric space. Then it follows that $\mathscr{B}_{q}(X)$ is equivalent to $\mathscr{B}_{q^{-1}}(X)$.

Proof. Since any subset $A$ of $X$ is $q$-bounded if and only if it is $q^{-1}$-bounded.

It is well-known that any quasi-pseudometric space $(X, q)$ can be seen as a bitopological space $\left(X, \tau(q), \tau\left(q^{-1}\right)\right)$. This motivates the following definition that we translate from [9] to our context.

Definition 4.7. (compare [9, Definition 1.4]) Let $(X, q)$ be a quasi-pseudometric space and $\mathscr{B}$ be a bornology on $X$. Then $(X, q, \mathscr{B})$ is a bornological bi-universe.

In the above definition if $q=q^{-1}$, then $(X, q, \mathscr{B})$ is bornological universe in the sense of [3].

Definition 4.8. (compare [9, Definition 1.7]) A bornological bi-universe $(X, q, \mathscr{B})$ is called quasi-metrizable if $\mathscr{\mathscr { B }}=\mathscr{\mathscr { B }}_{q}(X)$.

Definition 4.9. Let $(X, q)$ be a quasi-metric space. Then $q$ is said to induce a bornological bi-universe $(X, q, \mathscr{B})$ if $\mathscr{B}=\mathscr{B}_{q}(X)$.

Remark 4.10. Let $(X, q)$ be a quasi-metric space. Note that the bornological bi-universe $(X, q, \mathscr{B})$ is quasi-metrizable if and only if the bornological bi-universe $\left(X, q^{-1}, \mathscr{B}\right)$ is quasi-metrizable.

Example 4.11. [9] Consider the real line $\mathbb{R}$. Then

$$
U B(\mathbb{R})=\{A \subseteq \mathbb{R}: \text { there exists } r \in \mathbb{R} \text { such that } A \subseteq(-\infty, r)\}
$$

and

$$
L B(\mathbb{R})=\{A \subseteq \mathbb{R}: \text { there exists } r \in \mathbb{R} \text { such that } A \subseteq(r,+\infty)\}
$$

are bornologies in $\mathbb{R}$.

The topology $u=\{\emptyset, \mathbb{R}\} \cup\{(-\infty, a): a \in \mathbb{R}\}$ in $\mathbb{R}$ is called the upper topology and the topology $l=\{\emptyset, \mathbb{R}\} \cup\{(a,+\infty): a \in \mathbb{R}\}$ in $\mathbb{R}$ is called the lower topology.

Definition 4.12. (compare $\left[9\right.$, Definition 4.1]) Let $\left(X, q_{X}\right)$ and $\left(Y, q_{Y}\right)$ two quasi-metric spaces. A map $f: X \rightarrow Y$ is called bicontinuous with respect to $\left(\tau\left(q_{X}\right), \tau\left(q_{X}^{-1}\right), \tau\left(q_{Y}\right), \tau\left(q_{Y}^{-1}\right)\right)$ (in short bicontinuous) if

$$
\left\{f^{-1}(V): V \in \tau\left(q_{Y}\right)\right\} \subseteq \tau\left(q_{X}\right)
$$

and

$$
\left\{f^{-1}(V): V \in \tau\left(q_{Y}^{-1}\right)\right\} \subseteq \tau\left(q_{X}^{-1}\right) .
$$

Definition 4.13. (compare [9, Definition 4.2]) Let $(X, q)$ be a quasi-metric space. Then a $q$-characteristic function for a bornology $\mathscr{B}$ on $X$, is a bicontinuous function $f: X \rightarrow$ $[0,+\infty)$ with respect to $\left(\tau(q), \tau\left(q^{-1}\right), u, l\right)$ such that $\mathscr{B}=\{A \subseteq X: \sup \{f(x): x \in A\}<$ $+\infty\}$.

Example 4.14. (compare [9, Fact 4.3]) Let $(X, q)$ be a quasi-metric space and $a \in X$. Then the function $f: X \rightarrow[0,+\infty)$ defined by $f(x)=q(a, x)$ whenever $x \in X$ is bicontinuous. 
Lemma 4.15. (compare [9, Proposition 4.5]) Let $(X, q)$ be a quasi-metric space. If bornological bi-universe $(X, q, \mathscr{B})$ is quasi-metrizable, then there exists q-characteristic function for the bornology $\mathscr{B}$.

Definition 4.16. Let $(X, q)$ be a quasi-metric space. A bornology $\mathscr{B}$ on $X$ will be called $q$-proper if for each $A \in \mathscr{B}$, there exists $B \in \mathscr{B}$ such that $\mathrm{cl}_{\tau\left(q^{-1}\right)}(A) \subseteq \operatorname{int}_{\tau(q)}(B)$.

Remark 4.17. From [9, Proposition 4.6], it follows that if $(X, q, \mathscr{B})$ is a bornological bi-universe such that $\mathscr{\mathscr { B }}$ has a $q$-characteristic function, then $\mathscr{\mathscr { B }}$ is $q$-proper and second countable.

The following important theorem proved in [9] extends the well-known Hu's Theorem (see [3]) from metric point of view to quasi-metric settings.

Theorem 4.18. (compare [9, Theorem 4.7]) Let $(X, q)$ be a quasi-metric space. If $\mathscr{B}$ is a bornology on $X$, then the following conditions are equivalent:

(a) the bornological bi-universe respect to $\mathscr{B}$ is quasi-metrizable;

(b) there exists a q-characteristic function for $\mathscr{B}$;

(c) $\mathscr{B}$ is q-proper and it has countable base.

\section{Bornology on extended quasi-metric spaces}

Let $X$ be a set. If $\mathscr{A} \subseteq \mathcal{P}(X)$, then $\downarrow \mathscr{A}$ and $\sum \mathscr{A}$ are defined by

$$
\downarrow \mathscr{A}:=\{B \in \mathcal{P}(X): B \subseteq A \text { for some } A \in \mathscr{A}\}
$$

and

$$
\sum \mathscr{A}:=\left\{\bigcup_{i=1}^{n} A_{i}: A_{i} \in \mathscr{A} \text { whenever } i \in\{1, \cdots, n\} \text { with } n \in \mathbb{N}\right\} .
$$

If $\mathscr{B}$ is a bornology on $X$, then we say that a family $\mathscr{B}$ of subsets of $X$ is a base of $\mathscr{B}$ if $\downarrow(\mathscr{B})=\mathscr{B}$.

Definition 5.1. Let $\langle X, q\rangle$ be an extended quasi-metric space and $A$ be a subset of $X$. We say that $A$ is $\tau(q)$-relatively compact if $A$ is $\tau\left(q^{s}\right)$-relatively compact.

Note that the family of $\tau(q)$-relatively compact subsets of $X$ is a natural bornology on $\langle X, q\rangle$.

Lemma 5.2. (compare [1, Lemma 2.1]) Let $\langle X, q\rangle$ be a $T_{0}$-extended quasi-metric space. Then

(a) the family of all finite union of double open balls forms a base for $\mathscr{B}_{q}(X)$;

(b) $\mathscr{B}_{q}(X)$ contains the bornology of $\tau(q)$-relatively compact subsets of $X$;

(c) if the sequence $\left(x_{n}\right)$ is q-convergent and $q^{-1}$-convergent on $X$, then $\left\{x_{n}: n \in \mathbb{N}\right\} \in$ $\mathscr{B}_{q}(X)$.

Proof. (a) Consider

$$
\mathscr{F}_{B}=\left\{\bigcup_{k=1}^{n} B_{q}\left(x_{k}, r_{k}\right) \cap B_{q^{-1}}\left(x_{k}, s_{k}\right): x_{k} \in X \text { and } r_{k}, s_{k} \in(0, \infty) \text { whenever } n \in \mathbb{N}\right\} \text {. }
$$

We show that $\downarrow\left(\mathscr{F}_{B}\right)=\mathscr{O}_{q}(X)$.

Let $A \in \mathscr{F}_{B}$. Then $A=\bigcup_{k=1}^{n} B_{q}\left(x_{k}, r_{k}\right) \cap B_{q^{-1}}\left(x_{k}, s_{k}\right)$. Therefore $A \in \mathscr{B}_{q}(X)$ as $A$ is $q$-bounded. So $\downarrow\left(\mathscr{F}_{B}\right) \subseteq \mathscr{B}_{q}(X)$. 
Conversely, if $B \in \mathscr{B}_{q}(X)$, then there exists $x \in X$ and $r, s>0$ such that $B \subseteq$ $B_{q}(x, r) \cap B_{q^{-1}}(x, s)$. Since $B_{q}(x, r) \cap B_{q^{-1}}(x, s) \in \mathscr{F}_{B}$, it follows that $B \in \downarrow\left(\mathscr{F}_{B}\right)$.

(b) Let $A$ be a $\tau(q)$-relatively compact subset of $X$. Then by Definition 5.1, $A$ is $\tau\left(q^{s}\right)$ bounded. Hence $A \in \mathscr{\mathscr { B }}_{q}(X)$ by Remark 4.2.

(c) Suppose that the sequence $\left(x_{n}\right)$ is $q$-convergent to $x \in X$. Then, for $\epsilon=1$, there exists $N^{\prime} \in \mathbb{N}$ such that $q\left(x_{n}, x\right)<\epsilon$ whenever $n \geq N^{\prime}$.

If $r=\max \left\{1, q\left(x_{0}, x\right), q\left(x_{1}, x\right), \cdots, q\left(x_{N^{\prime}}, x\right)\right\}$, then $q\left(x_{n}, x\right)<r$ whenever $n \in \mathbb{N}$.

By similar arguments, if $\left(x_{n}\right)$ is $q^{-1}$-convergent to $x \in X$, then $q\left(x, x_{n}\right)<s$ whenever $n \in \mathbb{N}$. Therefore, $x_{n} \in B_{q}(x, r) \cap B_{q^{-1}}(x, s)$ whenever $n \in \mathbb{N}$.

Theorem 5.3. Let $\langle X, q\rangle$ be a $T_{0}$-extended quasi-metric space and let $\mathscr{B}$ be a bornology on $X$. Then $\mathscr{B}=\mathscr{\mathscr { B }}_{q}(X)$ if and only if there exist $\mathscr{A} \subseteq \mathscr{B}$ such that $\downarrow\left(\sum(\mathscr{A})\right)=\mathscr{B}$ and a partition $\left\{\mathscr{A}_{i}: i \in I\right\}$ of $\mathscr{A}$ with the following properties:

(1) $\mathscr{A}_{i}$ contains a nonempty subset of $X$ whenever $i \in I$;

(2) for all $A_{1} \in \mathscr{A}$, there exists $A_{2} \in \mathscr{A}$ i with $\operatorname{cl}_{\tau\left(q^{-1}\right)}\left(A_{1}\right) \subseteq \operatorname{int}_{\tau(q)}\left(A_{2}\right)$ whenever $i \in I$;

(3) whenever $A_{i} \in \mathscr{A} i$ and $A_{j} \in \mathscr{C}$ for $i \neq j$, then $A_{i} \cap A_{j}=\emptyset$;

(4) each $\mathscr{A}_{i}$ has a countable subfamily which is cofinal in $\mathscr{A}_{i}$ with respect to inclusion.

Proof. Suppose $q$ is a $T_{0}$-extended quasi-metric on $X$ and $\mathscr{B}=\mathscr{B}_{q}(X)$.

Let $\left\{X_{i}: i \in I\right\}$ be quasi-metric components of $X$,

$$
\mathscr{A}=\left\{B_{q}(y, r) \cap B_{q^{-1}}(y, s): y \in X_{i} \text { and } s, r>0\right\}
$$

and $\mathscr{A}$ be the collection of double open balls in $X$. Then by Lemma 5.2 (a)

$$
\sum\left(\bigcup_{i \in I} \mathscr{\mathscr { C } _ { i }}\right)=\sum\left(\bigcup_{i \in I}\left\{B_{q}(y, r) \cap B_{q^{-1}}(y, s): y \in X_{i} \text { and } s, r>0\right\}\right)
$$

and $\left\{\mathscr{A}_{i}: i \in I\right\}$ is a partition of $\mathscr{A}$. Thus, we have

$$
\mathscr{O}_{q}(X)=\downarrow\left(\sum\left(\bigcup_{i \in I} \mathscr{\mathscr { A }}\right)\right)=\downarrow\left(\sum(\mathscr{A})\right) .
$$

If $A \in \mathscr{A}$ whenever $i \in I$, then $A$ is double open ball. Therefore $A \neq \emptyset$ and the property (1) holds.

Moreover, since $\mathscr{A} /$ is a bornology on $X_{i}$ whenever $i \in I$, then by Theorem $4.18 \mathscr{C}$ is $q_{i}$-proper and has a countable base. Therefore, properties (2), (3) and (4) hold.

Conversely, suppose that there exists $\mathscr{A}$ with $\mathscr{A} \subseteq \mathscr{B}$ such that $\downarrow\left(\sum(\mathscr{A})\right)=\mathscr{B}$ for some $\mathscr{A}$ with $\{\mathscr{A}: i \in I\}$ is a partition of $\mathscr{A}$, where $\mathscr{A}$ satisfies properties (1), (2), (3) and (4).

Let $X_{i}=\bigcup_{i \in I} \mathscr{A}$. Then $X_{i} \neq \emptyset$ since the family $\mathscr{A} /$ contains a nonempty subset of $X$ whenever $i \in I$.

Furthermore, by property (2) we have that $X_{i}$ is $\tau(q)$-open whenever $i \in I$. Moreover, from property (3) $\left\{X_{i}: i \in I\right\}$ is a pairwise disjoint family. Then we have that $X_{i}$ is $\tau\left(q^{1}\right)$-closed and $\tau(q)$-open whenever $i \in I$. 


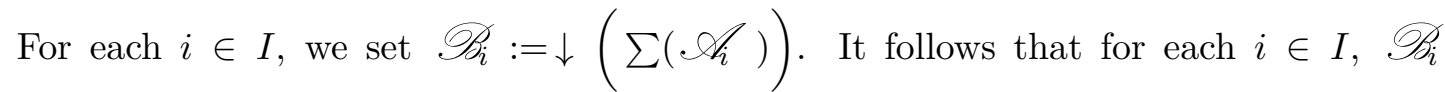

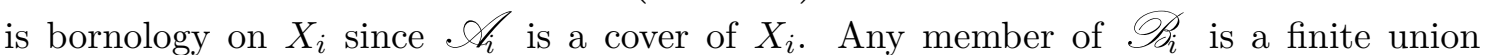
of elements of $\mathscr{C} /$ and since the $\tau\left(q^{-1}\right)$-closure of a finite union is the union of $\tau\left(q^{-1}\right)$ closures, then family $\mathscr{B}_{i}$ satisfies the property (2) whenever $i \in I$. Similarly, the family $\mathscr{S}_{i}$ satisfies the property (3) whenever $i \in I$.

Finally, if $\mathscr{C}_{i}$ is a countable and cofinal family in $\mathscr{C} i$ whenever $i \in I$, then $\sum\left(\mathscr{C}_{i}\right)$ is countable and cofinal in $\mathscr{\mathscr { B }}_{i}$. That is whenever $i \in I$, the family $\mathscr{B}_{i}$ satisfies the property (4). Then by Theorem 4.18, there exists a quasi-metric $q_{i}$ on $X_{i}$ such that $\mathscr{\mathscr { B }}_{i}=\mathscr{\mathscr { B }}_{q_{i}}\left(X_{i}\right)$.

Since $\downarrow\left(\sum(\mathscr{A})\right)$ is assumed to be a cover of $X$, we conclude that $\mathscr{A}$ is a cover of $X$ and so is $\left\{X_{i}: i \in I\right\}$ a cover of $X$. So we can define $q: X \times X \rightarrow[0, \infty]$ by

$$
q(x, y)= \begin{cases}q_{i}(x, y), & \text { there exists } i \in I \text { with }\{x, y\} \subseteq X_{i} \\ \infty, & \text { otherwise. }\end{cases}
$$

Therefore, we have

$$
\downarrow\left(\sum\left(\bigcup_{i \in I} \mathscr{\mathscr { C }}\right)\right)=\downarrow\left(\sum(\mathscr{A})\right)=\mathscr{\mathscr { B }}
$$

Since any member of $\mathscr{\mathscr { B }}_{i}$ is a finite union of elements of $\mathscr{\mathscr { A } _ { i }}$, with $q$ defined above, we have

$$
\mathscr{B}_{q}(X)=\sum\left(\bigcup_{i \in I} \mathscr{\mathscr { B }}_{i}\right)=\downarrow\left(\sum\left(\bigcup_{i \in I} \mathscr{A}_{i}\right)\right)
$$

Combining (5.1) and (5.2), we have

$$
\mathscr{B}(X)=\mathscr{B}
$$

Theorem 5.4. (compare [1, Theorem 4.3]) Let $\langle X, q\rangle$ be a $T_{0}$-extended quasi-metric space. Then the set of quasi-metric components induced by $q$ is countable if and only if there exists a compatible quasi-metric $q^{\prime}$ such that $\mathscr{\mathscr { B }}_{q}(X)=\mathscr{\mathscr { B }}_{q^{\prime}}(X)$.

Proof. Let $I$ be a countable set and $\left(x_{i}\right)_{i \in I}$ be a family of points in $X$. Suppose $\left\{\mathrm{qmc}_{q}\left(x_{i}\right): i \in I\right\}$ is the set of distinct quasi-metric components induced by $q$. Then $\sum\left(\left\{B_{q}\left(x_{i}, n\right) \cap B_{q^{-1}}\left(x_{i}, m\right): i \in I, n, m \in \mathbb{N}\right\}\right)$ is a countable base for $\mathscr{B}_{q}(X)$ and for each $A \in \mathscr{\mathscr { B }}_{q}(X)$, there exists $B \in \mathscr{\mathscr { B }}_{q}(X)$ with $\mathrm{cl}_{\tau\left(q^{-1}\right)}(A) \subseteq \operatorname{int}_{\tau(q)}(B)$. Then by Theorem 4.18, there exists a compatible quasi-metric $q^{\prime}=\min \{1, q\}$ such that $\mathscr{B}_{q}(X)=\mathscr{\mathscr { B }}_{q^{\prime}}(X)$.

Conversely, suppose that there exists a compatible quasi-metric $q^{\prime}$ such that $\mathscr{\mathscr { B }}_{q}(X)=$ $\mathscr{B}_{q^{\prime}}(X)$. Then by Theorem 4.18, $\mathscr{B}_{q}(X)$ has a countable base and hence $\sum\left(\left\{B_{q}\left(x_{i}, n\right) \cap\right.\right.$ $\left.\left.B_{q^{-1}}\left(x_{i}, m\right): i \in I, n, m \in \mathbb{N}\right\}\right)$ contains a countable cofinal family within $\mathscr{\mathscr { B }}_{q}(X)$. It follows that $I$ is countable as $\left\{\left\{x_{i}\right\}: i \in I\right\}$ is a family of $q$-bounded sets.

Acknowledgment. The authors would like to thank the referee for her/his suggestions and comments that have clearly improved the presentation of this article.

\section{References}

[1] G. Beer, The structure of extended Real-valued metric spaces, Set-Valued Var. Anal 21 (4), 591-602, 2013.

[2] P. Fletcherand and W.F. Lindgren, Quasi-uniform spaces (Dekker, 1982). 
[3] S.T. Hu, Boundedness in a topological space, J. Math. Pures Appl. 228, 287-320, 1949.

[4] K. Kemajou, H.P. Künzi and O. Olela Otafudu, The Isbell-hull of a di-space, Topol Appl. 159 (9), 2463-2475, 2012.

[5] H.P. Künzi, An introduction to quasi-uniform spaces, Contemp. Math. 486, 239-304, 2009.

[6] H.P. Künzi and C. Makitu Kivuvu, A double completion for an arbitrary $T_{0}$-quasimetric space, J. Log. Algebr. Program. 76 (2), 251-269, 2008.

[7] H.P. Künzi and O. Olela Otafudu, q-hyperconvexity in quasipseudometric spaces and fixed point theorems, J. Funct. Spaces Appl. Art.ID 765903, 18 pp., 2012,

[8] M.G. Murdeshwar and K.K. Theckedath, Boundedness in a quasi-uniform space, Canad. Math. Bull. 13, 367-370, 1970.

[9] A. Piekosz and E. Wajch, Quasi-metrizability of bornological biuniverses in ZF, J. Convex Anal. 22 (4), 1041-1060, 2015. 